\title{
Correspondence
}

\section{Gastric and jejunal feeding in high risk neonates}

Sir,

Many intensive care nurseries have used transpyloric feeding since it was introduced by Rhea and Kilby ${ }^{1}$ and in several studies ${ }^{2-3}$ nasogastric and nasojejunal methods have been compared but the results have been mainly qualitative. Little quantitative information is available on digestion or absorption of nutrients by these infants. Roy et al. $^{4}$ made such comparisons but rejected sick infants from their study. In practice however, it is the sick infant who is most likely to benefit from tube feeding. We therefore studied nasogastric versus nasojejunal feeding in high risk, newborn babies.

Six premature infants (birthweights, 820 to $1400 \mathrm{~g}$; gestational ages, 27 to 32 weeks) were studied in the second to fourth week of life. All except 1 had severe respiratory distress, 2 had intraventricular haemorrhages, and 1 had group B streptococcal septicaemia. Three babies began the study with nasogastric and 3 with nasojejunal feeding. Each regimen was followed for a week and then the other regimen was followed. Three babies received expressed breast milk and 2 received Cow and Gate's Premium Formula throughout the study. One baby received expressed breast milk for the first week and Premium Formula for the next 2 weeks. Stools were collected and pooled during the last 3 days of each week. The total nitrogen and fatty acid content of the stools and the expressed breast milk were determined by analysis. Comparison of the feeding methods showed no difference in volume of milk received, weight gain, or frequency of stools, and there was no consistent difference in nutrient absorption. Clinical observations suggested a tendency for the development of apnoeic attacks and abdominal distension during nasogastric feeding.

Wide variations in fat (12 to $96 \%$ ) and nitrogen ( 22 to 93\%) absorption were observed but these seemed to be related to the clinical condition and postnatal age of the baby rather than to the feeding method. In particular, there was some evidence that fat absorption increased with age, coinciding with a general clinical improvement. Fat absorption is known to be greater in term than in premature infants, ${ }^{5}$ and this trend continues in early postnatal development.

Absorption of nutrients by premature babies is influenced by many factors, and any study on the efficacy of different feeding methods must take this into account. Improvements in fat and nitrogen absorption by nasogastric compared with nasojejunal feeding have been reported for healthy, low birthweight infants but these cannot necessarily be expected to apply to the very sick neonate; in our study nutrient absorption was often extremely low. Any decision about the best type of feeding regimen therefore, ought to be made from clinical rather than from nutritional considerations until the results of further studies become available.

\section{References}

1 Rhea J W, Kilby J O. A nasojejunal tube for infant feeding. Pediatrics 1970; 46: 36-40.

2 Van Caillie M, Powell K G. Nasoduodenal versus nasogastric feeding in the very low birthweight infant. Pediatrics 1975; 56: 1065-72.

3 Wolfsdorf J, Makarawa S, Fernandes C, Fenner A. Transpyloric feeding in small preterm infants. Arch Dis Child 1975; 50: 723-6.

4 Roy N R, Pollnitz R P, Hamilton J R, Chance G W. Impaired assimilation of nasojejunal feeds in healthy low birthweight newborn infants. J Pediatr 1977; 90: 431-4.

5 Widdowson E M. Absorption and excretion of fat, nitrogen, and minerals from 'filled' milks by babies one week old. Lancet 1965; ii : 1099-105.

M de Carvalho and J A Davis Department of Paediatrics, Level 8, Addenbrookes's Hospital, Hills Road, Cambridge CB2 $2 Q Q$

G J Hudson AND W A Coward Medical Research Council, Dunn Nutrition Unit, Milton Road, Cambridge CB4 $1 \mathrm{XJ}$

\section{Diagnosis of coeliac disease}

Sir,

McNeish and his colleagues, ${ }^{1}$ in presenting results of the ESPGAN questionnaire, mentioned 20 patients in whom mucosal relapse after reintroduction of gluten-containing diets took more than 2 years to appear. Details were available on 7 of these 20 patients, but this was regarded as insufficient. We can now provide information on 1 of the 7 children; this has an important bearing on the '2-year rule', which implies that if the mucosa is still normal after 2 years on a normal diet (in a child whose illness originally responded to a gluten-free diet) the original illness was not coeliac disease (gluten enteropathy).

This girl, described in detail, ${ }^{2-3}$ was found to have a flat mucosa at 16 months and spent 57 months on a gluten-free diet with mucosal remission. After 28 months on a gluten-containing diet the mucosa was still normal on histology and she had normal mucosal lactase, sucrase, alkaline phosphatase, and interepithelial lymphocyte 